\title{
Análisis De La Gestión De Un Dispositivo Didáctico Formulado En EI Marco De La TAD
}

\author{
Analysis Of The Management Of A Didactic Device Formulated In The \\ Framework Of The TAD
}

\author{
Ana Rosa Corica* \\ Consejo Nacional de Investigaciones Científicas y Técnicas (CONICET) \\ NIECyT- UNCPBA
}

\begin{abstract}
Resumen
En este trabajo se describen resultados de la gestión de un dispositivo didáctico por un estudiante de profesorado en matemática. La implementación se realizó en un curso de quinto año de la escuela secundaria Argentina. La propuesta didáctica posibilitó la realización de algunos gestos propios del paradigma del cuestionamiento del mundo en las condiciones actuales de la escuela secundaria. En este trabajo se profundiza en los resultados del estudio de la última situación propuesta, que tiene como objetivo evaluar el estudio realizado.
\end{abstract}

Palabras clave: Matemática; Teoría Antropológica de lo Didáctico; Escuela secundaria

\begin{abstract}
This paper describes the results of the management of a didactic device by a mathematics trainee. The implementation was made in a fifth year of the Argentine high school. The didactic proposal made possible the realization of some gestures of the world question paradigm in the current conditions of the high school. In this paper, the study results of the last proposed situation are deepened. This situation has as aim to assessment the study carried out.
\end{abstract}

Keywords: Mathematics; Anthropological Theory of the Didactical; High School

\section{Introducción}

El paradigma dominante en los sistemas escolares para la enseñanza de la matemática se encuentra gobernado por el paradigma de la visita de obras (PVO) (Chevallard, 2006). Su

\footnotetext{
* Doctora en Ciencias de la Educación por la UNC, Profesora en Matemática y Física y Licenciada en Educación Matemática por la UNCPBA. Investigador Adjunto del CONICET, Profesor Adjunto de la Facultad de Ciencias Exactas de la UNCPBA, Tandil, Buenos Aires, Argentina. E-mail: acorica@exa.unicen.edu.ar.
} 
presencia genera que surjan fenómenos didácticos que fueron identificados por distintas investigaciones: el carácter puntual de las praxeologías matemáticas que se estudian, la tendencia a hacer algorítmicas las tareas matemática, la escasa incidencia del cuestionamiento tecnológico de las técnicas y del bloque tecnológico - teórico sobre la práctica matemática, la desarticulación entre las diferentes áreas y sectores de la matemática escolar, el autismo temático vinculado con el olvido de las posibles razones de ser de las praxeologías, la autosuficiencia de la matemática escolar que aparece encerrada en sí misma y cuya relación con el resto de disciplinas se reduce a aplicaciones (Barquero, 2009; Fonseca, 2004; Gascón, 2004; Oliveira, 2015; Serrano, 2013). En contra posición a este paradigma, Chevallard (2007) propone el paradigma del cuestionamiento del mundo (PCM). En esta perspectiva, la actividad matemática es más que resolver problemas: se trata de formular y responder peguntas, buscar en diferentes medias, desarrollar diferentes técnicas, realizar conjeturas, validar soluciones, interactuar con otros de la comunidad de estudio, cotejar resultados, técnicas, validaciones, etc.

Es requisito fundamental en esta propuesta, romper con la concepción atomizada de la matemática y posibilitar recorrer diversas praxeologías según las necesidades del estudio. En esta propuesta el profesor tiene que desplazarse del papel de protagonista principal que se acepta en las instituciones escolares y ceder lugar al hacer de los estudiantes. No se trata de un profesor ausente, sino de un director del proceso de estudio y de investigación, capaz de incidir oportuna y eficazmente para hacer evolucionar el estudio. Es necesario que sus ayudas al estudio se orienten a que la comunidad logre problematizar la situación de estudio y que el sentido de la misma no se pierda.

En los últimos años, la investigación didáctica en el marco de la Teoría Antropológica de lo Didáctico (TAD) dio lugar al diseñó de un número considerable de dispositivos didácticos compatibles con el PCM, que abarcan distintos ámbitos de la matemática y distintas etapas educativas (Barquero, 2009, Costa, 2013; Donvito, Otero y Sureda, 2014; Fonseca, 2010, 2011a, 2011b, 2011c; Fonseca, Pereira y Casas, 2010; Llanos 2012; Oliveira, 2015; Otero, Gazzola, Llanos y Arlego; 2016; Parra, 2013; Rodriguez, 2005; Ruiz-Munzón, 2010, Ruiz, Bosch y Gascón, 2007; Ruiz-Higueras y García García, 2011; Salgado, Otero y Parra, 2017; Serrano, Bosch, Gascón, 2010). Sin embargo, la transferencia de estos resultados al sistema educativo son escasas (Cid, Muñoz, Ruíz, 2018).

En este trabajo se reportan resultados de la gestión de un dispositivo didáctico compatible con el PCM y dirigido por un estudiante de profesorado en matemática (EPM). Se propone comparar tarifas de telefonía celular analizando el estado actual de compañías en 
Argentina. Esta situación genera un tipo de actividad matemática poco habitual en los sistemas actuales de enseñanza, donde la modelización del sistema cobra un lugar esencial. Esto implica un trabajo a largo plazo, con el objetivo de responder a una pregunta inicial que constituye su razón de ser. En particular, en este trabajo se profundiza en el análisis de la última situación que contempla el dispositivo didáctico. Esta tiene por finalidad evaluar el estudio realizado: lo que los estudiantes saben y lo que saben hacer en el marco del proyecto considerado. Para esto se propone observar los grupos realizando una tarea específica, donde la formulación de conjeturas y toma de decisiones resultan ser tareas cruciales.

\section{Marco Teórico}

La TAD propone introducir en los sistemas de enseñanza el PCM. Este tiene como objetivo educativo crear nuevas posturas hacia el aprendizaje caracterizadas por la actitud de problematización, asociada al carácter herbartiano, procognitivo y exotérico (Chevallard, 2013a). Esto es, herbartiano en el sentido de que el motor del aprendizaje es la actitud receptiva hacia la formulación de preguntas y problemas sin resolver; procognitivo en el sentido de considerar que el conocimiento está por descubrirse y no a la revisión del conocimiento ya descubierto; exotérico en el sentido de inmerso en el estudio porque siempre hay lugar para nuevos conocimiento sobre una disciplina; y finalmente la actitud de problematización se caracteriza por formular preguntas, tal que algunas se conviertan en problemas para al menos un grupo de personas.

El modelo didáctico general que emplea la TAD para describir la estructura de los procesos de estudio característicos de los diferentes paradigmas y la relación entre ellos es el esquema herbartiano (Chevallard, 2004). Así, en el caso extremo del PVO, el proceso de estudio puede ser descripto del siguiente modo: el profesor $y$ ha estudiado una obra $O$ y la presenta a los estudiantes de manera discursiva. Como toda obra, $O$ resulta ser el trabajo humano y, es una respuesta $R^{*}$ a determinadas cuestiones $Q_{y}$ que se encuentran ausentes en el discurso. El profesor construye el medio didáctico My que utiliza para elaborar $R^{*}$. De esta manera, el esquema herbartiano resulta ser: $\left[S(X, y, O) \rightarrow M_{y}\right] \rightarrow R^{\bullet}$. En el caso del PCM, las obras $O$ que se proponen estudiar se presentan en forma de cuestiones $Q$. El medio didáctico $M=$ $\left\{R_{i}^{\diamond}, O_{j}, Q_{k}, R_{k}\right\}$, está construido por la comunidad de estudio $[X, Y] \mathrm{y}$ contiene todas las herramientas necesarias para que esta construya una respuesta $R^{\bullet}$ a las cuestiones $Q$. El esquema herbartiano reducido expresa el proceso de estudio mediante: $[S(X, y, O) \rightarrow M] \rightarrow R^{*}$. 
La construcción de $R^{\boldsymbol{\nu}}$ es producto de un recorrido de estudio e investigación a lo largo del que se evalúa la relevancia de las respuestas $R_{i}^{\diamond}$ que la cultura proporciona a cuestiones próximas o relacionadas con $Q$. Se estudiarán obras de todo tipo $O_{j}$ potencialmente útiles para deconstruir y reconstruir dichas respuestas $R_{i}^{\diamond}$, para proporcionar respuestas provisionales $R_{k}$ a diversas cuestiones $Q_{k}$, derivadas de $Q$ y se utilizarán todos estos medios para construir, validar y difundir $R^{\star}$. La dinámica de los elementos que constituyen la estructura de estos procesos de estudio, se describe en términos de dialécticas (Chevallard, 2007, 2013b, 2013c):

- La dialéctica del estudio y de la investigación. Una investigación supone la combinación del estudio de preguntas y respuestas: no es posible investigar sin estudiar y a su vez un estudio genuino es productor de preguntas a ser investigadas.

- La dialéctica del individuo y el colectivo. Los estudiantes con su director de estudio acuerdan el conjunto de tareas a realizar y negociar las responsabilidades que debe asumir cada uno.

- Dialéctica del análisis-síntesis praxeológica y del análisis-sintesis didáctica. La construcción de una respuesta a una pregunta requiere concretar un análisis de esos saberes y determinar qué es lo útil, lo funcional para la construcción de la respuesta buscada.

- La dialéctica de entrar y salir-de tema. Si la pregunta es amplia y generativa, es necesario habilitar la posibilidad de salir del tema, incluso hasta puede ser necesario salir de la disciplina de referencia, y reingresar posteriormente.

- La dialéctica de las cajas negras y las cajas claras. Se refiere a la necesidad de establecer si una obra merece ser estudiada, aclarada, analizada, etc., o si ciertos saberes se dejarán en un nivel de gris. Es decir, saberes que no son esenciales para responder la cuestión generatriz o sus derivadas.

- La dialéctica del paracaidista y de las trufas. Se refiere a la condición de exploradores que asumen los actores del sistema didáctico. Tienen que tomar una gran distancia del problema y explorar el terreno desde muy arriba. Esta inspección difícilmente encuentra de inmediato lo que se busca, y requiere de gestos de acercamiento, para analizar la utilidad de lo encontrado. Esta dialéctica se opone al hábito escolar de la inmediatez en la búsqueda de soluciones, donde se estudian respuestas inmediatas y triviales.

- La dialéctica de los media y los medios. La elaboración de las sucesivas respuestas provisionales requiere de respuestas preestablecidas, accesibles a través de los 
diferentes medios de comunicación y difusión: los media. Estos pueden ser libros, artículos de investigación, apuntes de clase etc.

- La dialéctica de la lectura y la escritura. Hace referencia al proceso de evitar la transcripción formal de respuestas existentes. Se trata de tomar de ellas la parte útil y volver a escribirlas en notas de síntesis, glosarios etc.

- Dialéctica de la producción y la recepción: Es necesario difundir y defender la respuesta desarrollada por la comunidad de estudio. El saber no es importante por sí mismo, sino que sobreviene relevante debido a que la actividad matemática aporta respuestas consideradas valiosas por la comunidad de estudio.

\section{Metodología}

En este trabajo se propone una metodología de investigación cualitativa de corte exploratoria, descriptiva e interpretativa (Hernández, Fernández, Baptista, 2014). El dispositivo didáctico (DD) fue dirigido por un EPM que era un estudiantes de cuarto año de una carrera de profesorado en matemática de una Universidad Nacional Argentina, que experimentó un curso para la formación didáctico - matemática (Corica y Otero, 2016a, 2016b, 2017).

El DD que gestionó el EPM se inicia con el análisis de una situación que se ubica en comparar planes de telefonía celular. Esta fue desarrollada originariamente por Rodríguez (2005) en talleres diferenciados de la enseñanza habitual. Esta propuesta fue readaptada por Corica (2016) para ser gestionada en la escuela secundaria actual en Argentina, analizando el estado real de los planes que ofrecen diferentes compañías de telefonía celular.

La implementación del DD requirió de 11 sesiones (16 horas). En el inicio de cada sesión, el EPM solicitó a los estudiantes que recordaran lo que habían realizado la sesión anterior. De esta manera, los alumnos recuperaron qué preguntas estaban estudiando y qué respuestas estaban tratando de elaborar. Esto resultó relevante, pues los alumnos no se encontraban habituados al estudio de problemas donde su análisis no se finalice rápidamente (una o dos sesiones).

El investigador registró el audio general y realizó notas de campo de cada sesión. También recogió las producciones escritas de los estudiantes. Esto permitió que el EPM analizara con mayor detalle la producción de los estudiantes, reflexionara sobre su propia práctica y definiera cómo proseguir el estudio en la siguiente sesión. 


\subsection{Características Del Curso En El Que Se Desarrolló La Investigación}

La implementación se desarrolló en un curso de quinto año, en una escuela secundaria pública de gestión privada en Argentina. En el curso se destinan tres horas semanales para estudiar matemática, repartidas en un encuentro de dos horas y un encuentro de una hora. El curso estaba compuesto por $\mathrm{N}=35$ estudiantes cuyas edades oscilaban entre 15 y 16 años.

El grupo ha participado siempre de una enseñanza tradicional en matemática y en las demás disciplinas, pero los estudiantes mostraron interés en participar y explicar sus propuestas, más allá de que las técnicas empleadas fueran adecuadas o no. El EPM participó como ayudante del profesor del curso durante dos meses, antes de implementar el DD. Esto le permitió conocer al grupo, la forma en que estudiaban y contribuyó a que los estudiantes interactuaran con quien en poco tiempo sería su profesor.

\section{Características De La Gestión Del Dispositivo Didáctico}

En este apartado se describen las características esenciales del estudio realizado por la comunidad. El DD se inicia con el estudio de la cuestión $Q_{0}$ : En el mercado existen diversas compañias de telefonía celular y queremos contratar una, ¿Por cuál optar? ¿Por qué? Se trata de una pregunta que no tiene una respuesta final y requiere realizar conjeturas para poder establecer el plan que más conviene.

Para el estudio de $Q_{0}$, el EPM proporcionó material a los estudiantes acerca de los planes que ofrecen las 3 compañías de la que son usuarios. Esta información se encuentra disponible en internet, pero los estudiantes no contaban con conectividad para hacerlo. En general, los planes de las compañías de telefonía celular, contienen información acerca de costo de abono, megas disponibles para el uso de internet, números telefónicos para hablar de manera ilimitada, crédito disponible para todo consumo, SMS incluidos en el abono, costo para re-compra de internet, forma y costo de la tasación para llamada local y de larga distancia con el crédito disponible y luego de agotar el mismo, acceso a paquetes de música y video, minutos disponibles para hablar incluidos en el abono. En el Anexo 1 se sintetiza la principal información que ofrecen las compañías para cada plan en el momento en que el EPM implementó el DD.

A partir del análisis de la información, el EPM solicitó que los estudiantes formularan nuevas preguntas para ser estudiadas con posterioridad. De esta manera, se acordó estudiar las 
siguientes preguntas, expresadas por los estudiantes:

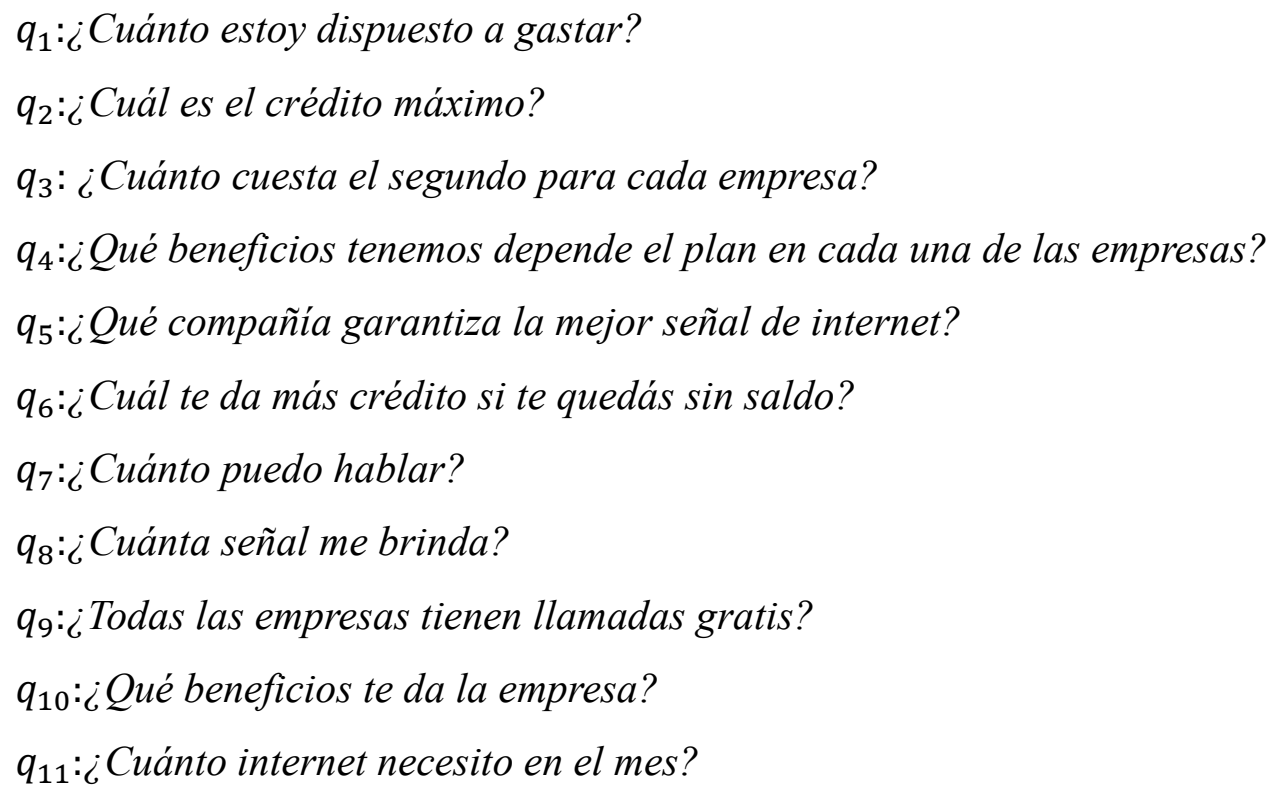

A continuación, los estudiantes y el EPM analizaron qué preguntas admitían respuesta inmediata, cuáles serían estudiadas y cuáles no. Uno de los aspectos que señalan los alumnos por qué elegir una compañía de telefonía celular es la calidad de la señal. Se acordó que las preguntas $q_{5}$ y $q_{8}$ no serían estudiadas, porque no contaban con suficientes herramientas para poder hacerlo. Sin embargo, mediante el empleo de Internet es posible conocer el área de cobertura de las compañías a partir del análisis de un mapa interactivo (por ejemplo en: www.claro.com.ar/empresas/institucional/cobertura/)

Las preguntas $q_{1}$ y $q_{11}$ resultan ser personales. La respuesta depende de las decisiones y uso que establezca realizar el usuario. Las preguntas $q_{4}, q_{6}, q_{9}$ y $q_{10}$ admiten respuesta inmediata al analizar el plan que ofrece cada compañía. Finalmente, el grupo acordó profundizar el estudio en $q_{2}, q_{3}$ y $q_{7}$. Si bien, $q_{2}$ parecería ser una pregunta que admite respuesta inmediata a partir del análisis del crédito disponible para todo consumo que ofrecen los planes de cada compañía, su análisis se torna problemático al ser estudiada en conjunto con $q_{3}$ у $q_{7}$.

Elaborar una respuesta para las preguntas $q_{2}$ y $q_{7}$ requiere comprender de qué manera las compañías de telefonía celular tasan las comunicaciones mediante llamadas. Al revisar los términos y condiciones legales que propone cada compañía, se concluye en lo siguiente: el primer bloque de 30 segundos tiene un costo fijo y luego de este período se comienza a tasar un costo por cada segundo que dure la comunicación. Por otro lado, la duración de las llamadas que se pueden efectuar se encuentra vinculada con el crédito disponible para todo consumo. 
Para maximizar el empleo de este crédito, es necesario establecer la comunicación y dejar que se agote el mismo. Si no sucede esto, cortar y volver a establecer la comunicación siempre se encuentra afectado por el costo del establecimiento de la llamada. La comunidad de estudio acordó que la expresión que modeliza la situación es:

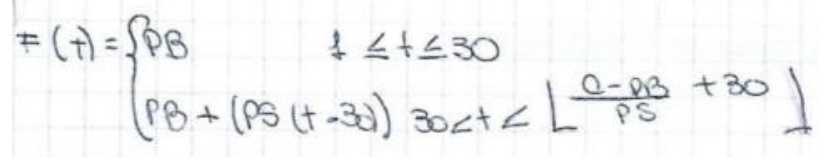

Imagen 1: Protocolo del Grupo 2

Fuente: una pesquisa

En esta expresión $P B$ indica el costo por establecimiento de la llamada, $P S$ el costo del segundo, $c$ el crédito para todo consumo y $t$ es el tiempo de duración de la llamada. La obtención de esta expresión es el resultado del trabajo conjunto entre los estudiantes y el EPM. Este último fue el encargado de introducir al medio nociones matemáticas desconocidas por los estudiantes, tales como: función definida a trozos y función piso.

Para el estudio de la cuestión: $q_{3}$ : ¿Cuánto cuesta el segundo para cada empresa? En principio, los estudiantes consideraban que el costo del segundo es el indicado por las compañías en la sección de términos y condiciones, haciendo de $q_{3}$ una pregunta que admite respuesta inmediata. Los estudiantes no lograban ingresar en la problemática de $q_{3}$, por lo que las intervenciones del EPM resultaron ser necesarias ayudas al estudio para que estos comprendieran cuál es el costo del segundo y de qué manera depende según el tiempo de duración de la comunicación. Los alumnos obtuvieron expresiones como la indicada por el Grupo 14.

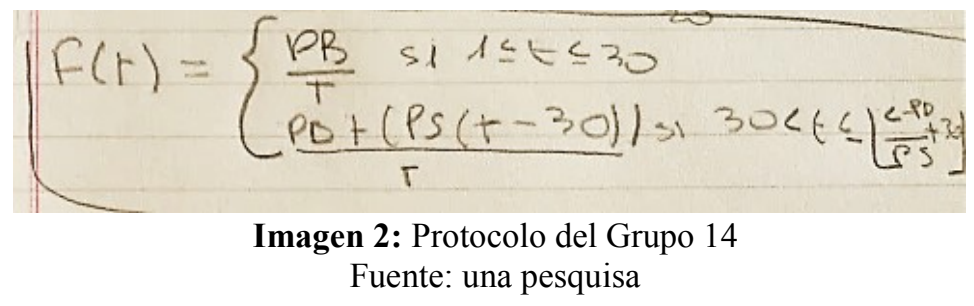

$\mathrm{F}(\mathrm{t})$ corresponde a la función costo medio, en la que las ramas se componen de funciones racionales. El EPM definió este tipo de función porque no había sido estudiado con anterioridad por los alumnos. Los estudiantes analizaron la expresión de la función, pudiendo concluir que cuando aumenta la duración de la llamada, el costo del segundo disminuye aproximándose al valor indicado por las compañías en la sección de términos y condiciones.

Durante el estudio, la matemática surgió como una necesidad para dar respuesta a la situación inicial y condujo a reencontrar la funcionalidad de algunas praxeologías estudiadas 
por los alumnos en años anteriores tales como: función constante, función lineal, estimación por redondeo y truncamiento, ecuaciones e inecuaciones lineales, dominio continuo. El estudio también exigió que los alumnos recorrieran nuevas praxeologías para ellos, relativas a: función racional, función definida a trozos, función techo, función piso, dominio discreto, función continua y discontinua. En particular, las nociones: función definida a trozos, función techo y función piso, no son propuestas para ser estudiadas en el diseño curricular de la escuela secundaria argentina.

En general, el estudio realizado generó gran interés en el grupo de estudiantes, porque remite a una situación actual y de interés social. El estudio fue rico en el conjunto de preguntas derivadas, transitando de esta manera uno de los gestos fundamentales de la pedagogía de la investigación: la dialéctica del estudio e investigación. La formulación de preguntas es de vital importancia en este tipo de estudio y el EPM logró que el grupo formulara y estudiara las propias. A pesar de que el análisis de las preguntas derivadas de $Q_{0}$ fue regulado por el EMP, los alumnos asumieron la responsabilidad de búsqueda de respuesta, proponiendo y estudiando situaciones concretas. Las respuestas fueron validadas por la propia experiencia y conocimientos de los estudiantes y por la constatación entre los grupos de trabajo y el EPM. Esto último se corresponde con uno de los gestos propios del PCM: la dialéctica del colectivo y el individuo. Esta fue transitada de manera parcial, porque el medio de estudio si bien fue gestado a partir de la propuesta de cada pequeño grupo, los estudiantes compartieron las mismas respuestas, recorriendo las mismas praxeologías.

Las ayudas al estudio ofrecidas por el EPM fueron fundamentales para avanzar y sostener la problematización de la situación inicial. Los estudiantes que participaron de la investigación, están habituados a una enseñanza tradicional e instantánea de la matemática, donde las tareas tienen respuestas únicas e inmediatas.

A continuación se presenta la situación final y se analiza la propuesta de los estudiantes. Con el análisis de esta situación se procuró evaluar el estudio realizado. Pues, durante las 10 primeras sesiones los alumnos analizaron sus propias preguntas no retomando la cuestión inicial $Q_{0}$ que dio origen al estudio.

\subsection{Análisis De La Situación Final Del Dispositivo Didáctico}

Durante el desarrollo de 10 sesiones el EPM gestionó el estudio de $Q_{0}$ y las cuestiones derivadas. En varias oportunidades el EPM tuvo la necesidad de distanciar el estudio del eje 
central de $Q_{0}$, y profundizar en el análisis de nociones matemáticas, para aportar respuesta a las preguntas: $q_{2}, q_{3}$ y $q_{7}$. En la última sesión se propuso el estudio de la situación que se indica a continuación. Esta recupera el sentido de $Q_{0}$ y permite que los estudiantes aporten respuesta sin intervenciones del profesor, luego de haber emprendido una vasta exploración de los planes que ofrecen las tres compañías consideradas.

\section{Situación final}

Un cliente quiere contratar una compañia de telefonía celular. Realizar un informe que le permita al cliente tomar decisiones teniendo en cuenta sus posibles necesidades.

En principio, las respuestas obtenidas eran escasas, por lo que se indicó a los estudiantes que consideren las siguientes preguntas y recomendaciones para redactar sus informes: ¿Qué compañía de telefonía móvil nos conviene contratar según nuestras necesidades? Por ejemplo: ¿Cuál es el crédito máximo? ¿Cuánto estoy dispuesto a pagar? ¿Utilizamos más los mensajes de textos que las llamadas? ¿Qué beneficios tiene cada empresa?, entre otras posibilidades. También se solicitó a los estudiantes que justificaran la propuesta teniendo en cuenta el estudio matemático realizado previamente.

Esta última tarea generó que la comunidad de estudio realizara conjeturas de potenciales clientes de telefonía celular y formulara posibles decisiones a tomar. La propuesta de los alumnos recuperó lo realizado desde el inicio del estudio, profundizando en la utilidad de la matemática para el análisis de la situación en aspectos que ellos consideraron pertinente. Se obtuvieron 17 trabajos correspondientes a la producción grupal de los estudiantes. Para el análisis de los protocolos se formularon las siguientes categorías de manera inductiva (Mejía, 2011):

- Sintetizar información. Esta categoría se refiere a la selección de información de cada plan, que se estima relevante para poder realizar comparaciones.

- Organizar datos en tabla. Indica los protocolos en los que los estudiantes construyen tablas de doble entrada para presentar la información que ofrecen las compañías y poder realizar comparaciones.

- Formular categorías. Recoge las producciones de los estudiantes en las que se explicitan categorías para establecer posibles usuarios de las compañías, y establecer qué plan es el que más conviene según las conjeturas que se realicen.

- Explicitar cálculos. Recoge las producciones en las que se explicitan resultados de cálculos para aumentar la información que ofrecen las compañías. 
- Explicitar las ecuaciones que permiten realizar los cálculos. Contempla la explicitación de la expresión, que permite obtener los resultados adicionados a la información brindada por las compañías.

- Extraer conclusión final. Recoge las propuestas en las que se indica una conclusión por lo que se considera una compañía mejor que otra.

En la Tabla 1, se registra con X la categoría que se identifica en cada trabajo. Estos son identificados según el número asignado a cada grupo.

Tabla 1: Categorías identificadas en los trabajos para la situación final

\begin{tabular}{|c|c|c|c|c|c|c|}
\hline Grupo & $\begin{array}{c}\text { Sintetizar } \\
\text { información }\end{array}$ & $\begin{array}{c}\text { Organizar } \\
\text { datos en } \\
\text { tabla }\end{array}$ & $\begin{array}{l}\text { Formular } \\
\text { categorías }\end{array}$ & $\begin{array}{l}\text { Explicitar } \\
\text { cálculos }\end{array}$ & $\begin{array}{l}\text { Explicitar las } \\
\text { ecuaciones } \\
\text { que permiten } \\
\text { realizar los } \\
\text { cálculos } \\
\text { matemáticos }\end{array}$ & $\begin{array}{c}\text { Extraer } \\
\text { conclusión } \\
\text { final }\end{array}$ \\
\hline 1 & $\mathbf{X}$ & $\mathbf{X}$ & $\mathbf{X}$ & $\mathbf{X}$ & & \\
\hline 2 & $\mathbf{X}$ & & $\mathbf{X}$ & $\mathbf{X}$ & & \\
\hline 3 & & & $\mathbf{X}$ & $\mathbf{X}$ & $\mathbf{X}$ & $\mathbf{X}$ \\
\hline 4 & $\mathbf{X}$ & & & $\mathbf{X}$ & $\mathbf{X}$ & $\mathbf{X}$ \\
\hline 5 & & & $\mathbf{X}$ & $\mathbf{X}$ & & \\
\hline 6 & $\mathbf{X}$ & $\mathbf{X}$ & & & & \\
\hline 7 & $\mathbf{X}$ & & $\mathbf{X}$ & $\mathbf{X}$ & $\mathbf{X}$ & $\mathbf{X}$ \\
\hline 8 & $\mathbf{X}$ & & $\mathbf{X}$ & & $\mathbf{X}$ & \\
\hline 9 & $\mathbf{X}$ & & $\mathbf{X}$ & & $\mathbf{X}$ & $\mathbf{X}$ \\
\hline 10 & $\mathbf{X}$ & & $\mathbf{X}$ & & $\mathbf{X}$ & \\
\hline 11 & & & $\mathbf{X}$ & & $\mathbf{X}$ & \\
\hline 12 & & & $\mathbf{X}$ & $\mathbf{X}$ & $\mathbf{X}$ & $\mathbf{X}$ \\
\hline 13 & $\mathbf{X}$ & & $\mathbf{X}$ & $\mathbf{X}$ & & $\mathbf{X}$ \\
\hline 14 & $\mathbf{X}$ & $\mathbf{X}$ & $\mathbf{X}$ & $\mathbf{X}$ & $\mathbf{X}$ & \\
\hline 15 & $\mathbf{X}$ & & $\mathbf{X}$ & & & $\mathbf{X}$ \\
\hline 16 & $\mathbf{X}$ & & $\mathbf{X}$ & $\mathbf{X}$ & $\mathbf{X}$ & $\mathbf{X}$ \\
\hline 17 & & & $\mathbf{X}$ & $\mathbf{X}$ & $\mathbf{X}$ & \\
\hline
\end{tabular}

Fuente: una pesquisa

A continuación se describen los resultados para las categorías que componen a la Tabla 1. En 12 trabajos, la información ofrecida por las compañías es reorganizada por los estudiantes (categorías: Sintetizar información y Organizar datos en tabla). No todos los casos contienen la misma información y disposición. En particular, se destaca la propuesta del Grupo 1, 6 y 14 donde la información es presentada en tablas de doble entrada. Por ejemplo, en la Imagen 3 se indica una tabla realizada por el Grupo 1. Esta se encuentra segmenta en tres partes (una para cada compañía seleccionada) y se establecen las siguientes categorías para comparar.

- Llamadas: Recoge información adicional a la ofrecida por las compañías. Se trata del resultado de un cálculo que es obtenido a partir de la fórmula como la indicada en la Imagen 1. Para su empleo se requiere interpretar la información brindada por las compañías. Aquí se 
indica el tiempo máximo de duración de una llamada empleando el crédito disponible para todo consumo.

- Internet: Indica los megas disponibles para ser empleado que contempla el plan.

- Saldo: Indica el crédito disponible para todo consumo ofrecido en el plan.

- Mensajes: Se refiere al número de mensajes disponibles para el envío sin costo contemplado en el plan.

- P. al mes: Indica el costo del plan.

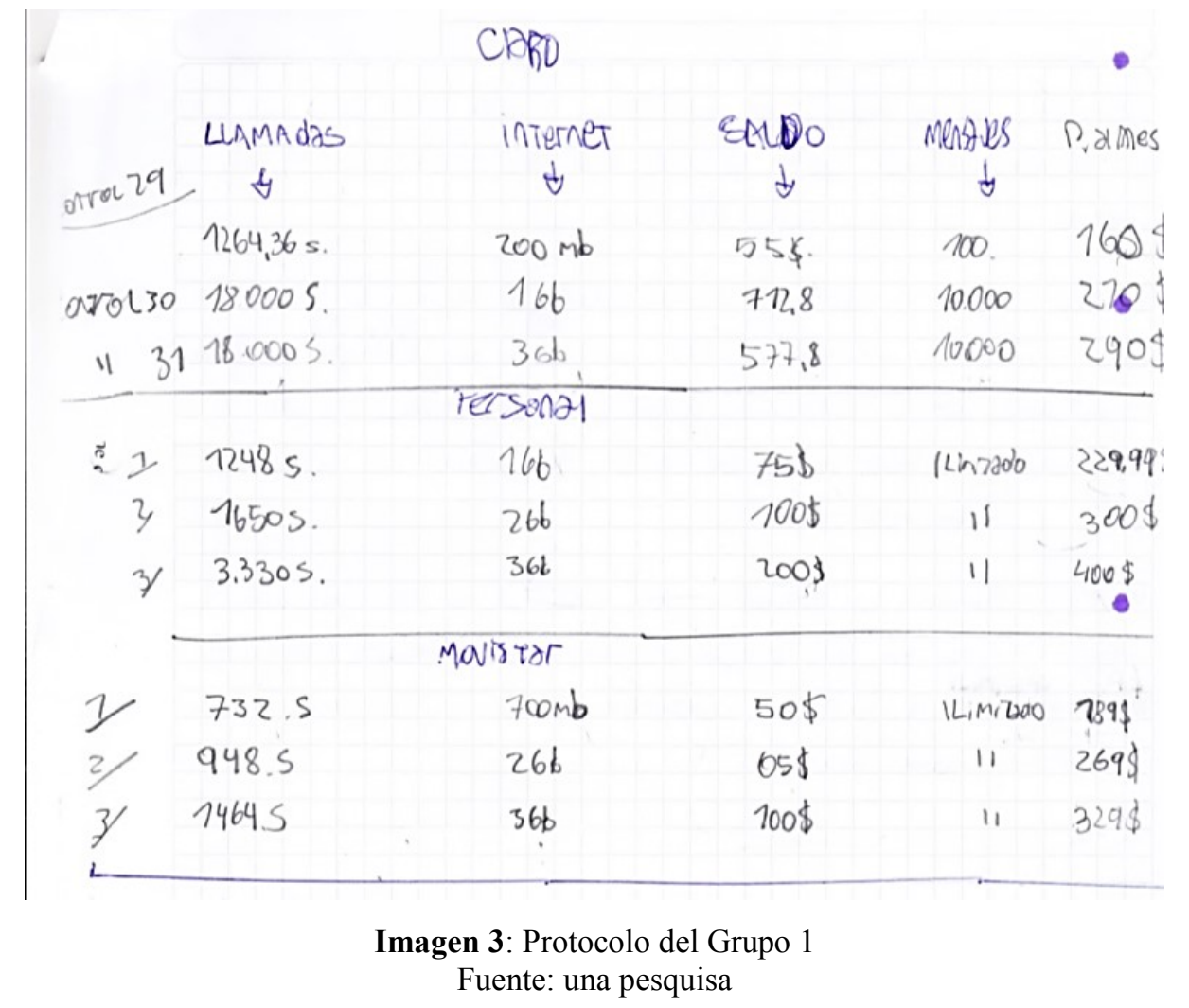

En este protocolo se observa que la necesidad de la matemática surge para confeccionar la primera columna (Llamadas) y la tercera columna (Saldo) de la tabla, con el propósito de ampliar la información brindada por las compañías. Con relación a la información indicada en la primera columna, resulta ser una información útil y necesaria para la extracción de conclusiones. Por ejemplo, para el plan Control 29 de la compañía Claro, si bien el costo del abono es más económico que los restantes, permite establecer comunicaciones mediante llamadas de aproximadamente igual duración que el plan más económico de Personal (1264 segundos para el plan Control 29 de Claro y 1248 segundos para el Plan 1 de Personal).

El cálculo del tiempo máximo posible para realizar llamadas, con el crédito disponible para todo consumo que ofrecen las compañías para cada plan, fue estudiado en sesiones anteriores. Sin embargo, los estudiantes del Grupo 1 ampliaron la información proporcionada 
por la compañía Claro: en la tercera columna, los estudiantes indican el equivalente en dinero de los 18000 segundos que ofrece la compañía Claro para sus planes (La compañía Claro para el Plan Control 30 y 31 incluyen 18000 segundos para llamadas, además de los \$30 para ser utilizados para todo consumo). Aquí la necesidad de la matemática emergió como respuesta a una inquietud de los estudiantes. En particular, en los cálculos realizados los alumnos olvidaron sumar los \$30 que cada plan ofrece para todo consumo.

En 9 trabajos la información es sintetizada haciendo más compleja la comparación. En la Imagen 4 se puede observar la propuesta del Grupo 2. Los estudiantes indican la información básica establecida en términos y condiciones para la compañía Movistar. En esta presentación también recuperan las fórmulas empleadas en sesiones previas que permite calcular el tiempo máximo de una llamada ideal con el crédito disponible para todo consumo. Aunque la unidad mínima de tiempo establecida para contabilizar la duración de una llamada es en segundos, los estudiantes la expresan en minutos. Esta propuesta es realizada para cada plan de cada compañía. Aquí la matemática toma lugar para ampliar la información acerca de cuánto tiempo puede durar como máximo una llamada según el plan que se contrate.

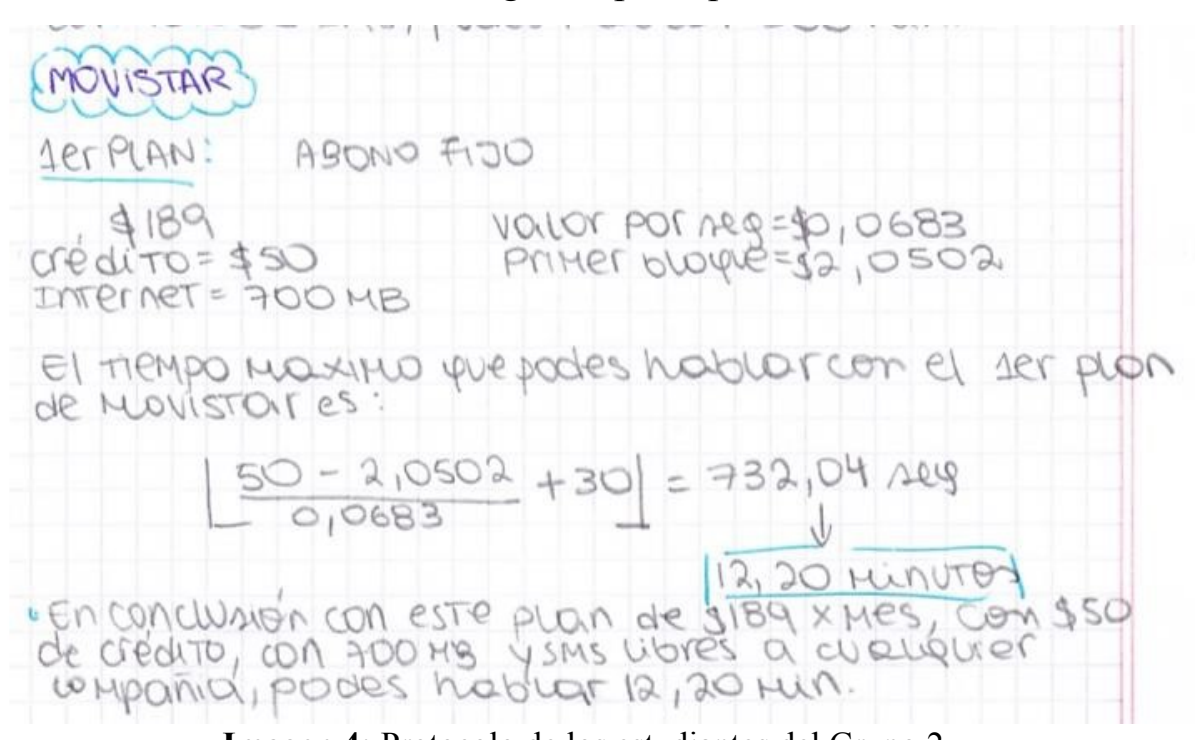

Imagen 4: Protocolo de los estudiantes del Grupo 2 Fuente: una pesquisa

En 15 trabajos se observa la formulación de categorías para poder establecer posibles usuarios de telefonía celular y determinar cuál es la más conveniente según el caso. Estas categorías se refieren a: Llamadas, internet, saldo, mensajes. En general, los estudiantes establecen un usuario representante de una de las categorías y en función de la información que se extrae de cada plan, se establece cuál conviene más. Esto se pone en evidencia en el protocolo del Grupo 1 (Imagen 5). 


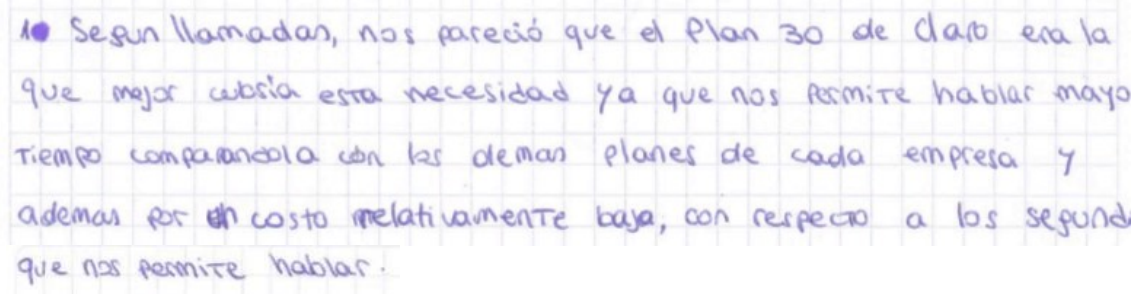

Imagen 5: Protocolo del Grupo 1

Fuente: una pesquisa

En esta propuesta solo se considera la categoría llamadas. Los estudiantes realizan la comparación contemplando toda la información que brinda la compañía para establecer la comunicación mediante llamadas, y empleando la matemática para ampliar la información, tal como se describió en el protocolo indicado en la Imagen 3.

Los estudiantes del Grupo 12 (Imagen 6) explicitan diferentes categorías de clientes. La propuesta va más allá de describir la información brindada por la compañía. Por ejemplo, los estudiantes extraen conclusiones en cuanto a la cantidad de mensajes ofrecidos por una de las compañías en relación al costo del abono:

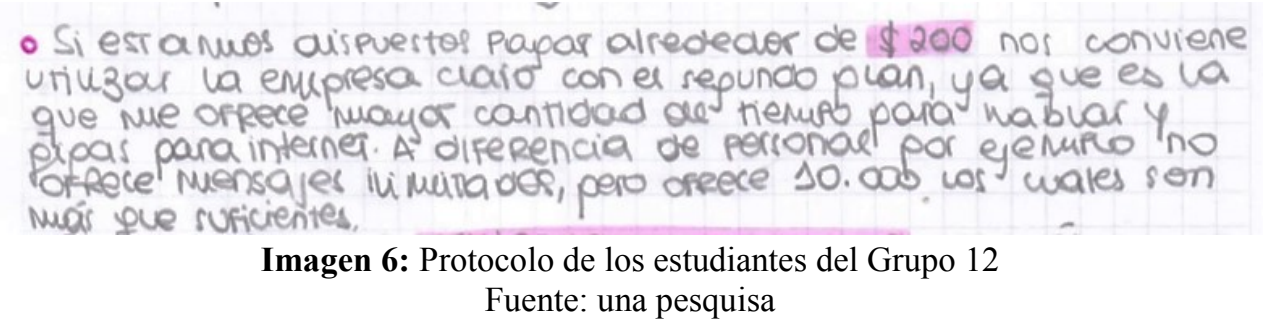

Así también, los estudiantes del Grupo 12 (Imagen 7) adicionan información que no se indica en la sección de términos y condiciones de las compañías y tampoco fue estudiado en sesiones anteriores. Es destacable esta propuesta, siendo que los estudiantes no se limitan a trabajar solo sobre lo estudiado en sesiones previas.

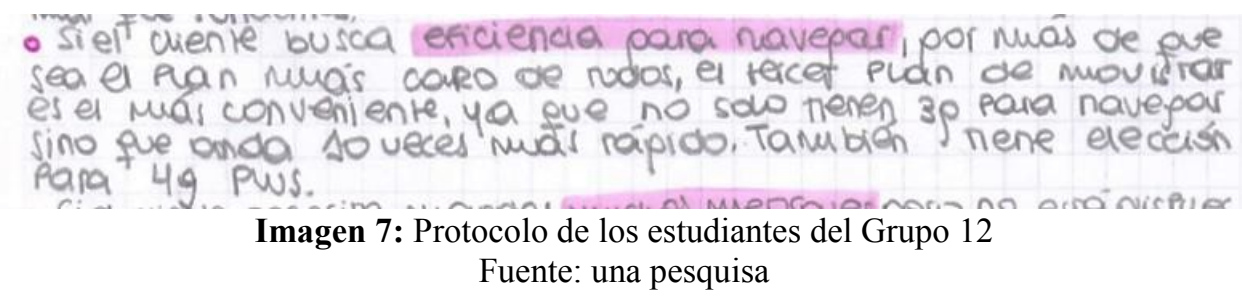

Finalmente, los estudiantes del Grupo 12 (Imagen 8) extraen una conclusión según el propio empleo que realizan de su compañía de telefonía celular.

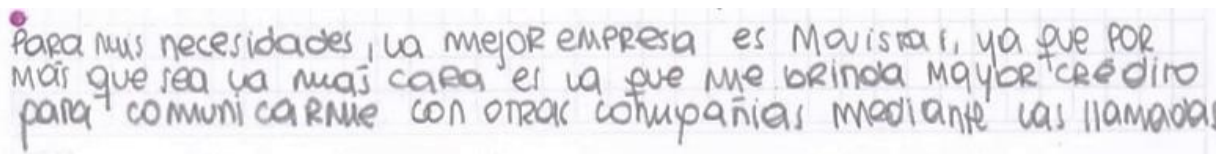

Imagen 8: Protocolo de los estudiantes del Grupo 12

Fuente: una pesquisa 
Con relación al empleo de la matemática, en 11 trabajos se recuperan las formulas construidas en sesiones anteriores. En 4 trabajos solo se indican resultados de cálculos sin explicitar los procedimientos para obtenerlos. En particular, se recupera la información necesaria para abordar la cuestión relativa a cuánto tiempo como máximo se puede establecer una comunicación mediante llamadas. Ninguno de los trabajos recupera la fórmula indicada en la Imagen 2. Durante el estudio de esta expresión la comunidad discutió el costo del segundo de las llamadas y su correlación con lo indicado en la sección de términos y condiciones. La ausencia de producciones en este sentido indica que los estudiantes no indagan acerca de para qué tiempo de duración de la llamada conviene un plan u otro.

\section{Conclusiones}

En este trabajo se presentaron resultados de la gestión de un DD que permite realizar algunos gestos propios de la PCM. La propuesta de la tarea final permitió que los estudiantes revisen lo realizado en sesiones previas y profundicen en lo que consideren pertinente.

La actitud de problematizar fue instalada por el EPM desde el inicio del estudio, pero no fue sostenida por los estudiantes sin su intervención. En las propuestas recogidas los estudiantes se limitan a establecer comparación de lo estudiado en sesiones previas sin indagar aspectos no estudiados. Por ejemplo, en sesiones previas se profundizó en la forma de facturación de llamadas de las compañías, pero no se indagó sobre aspectos específicos como las que se proponen para la compañía Movistar. Esta aplica un costo adicional según la distancia de la llamada y cuenta con otros beneficios como llamadas a telefonía fija sin costo. De esta manera los estudiantes podrían haber formulado y estudiado preguntas como ser: ¿Qué compañía conviene contratar si las llamadas son a larga distancia? ¿Cómo se facturan las llamadas de telefonía fija? ¿A partir de qué tiempo de duración de la llamada conviene un plan u otro? La exploración de información es superficial y sin salir de los límites que fueron estudiados en sesiones previas. Esto es atribuido a la formación previa de los estudiantes, quienes están habituados a una enseñanza tradicional e instantánea de la matemática, donde las tareas tienen respuestas únicas e inmediatas, y donde formular y responder sus propias preguntas no forma parte de sus responsabilidades.

En particular, el estudio de la última situación permitió que los alumnos empleen la matemática no como un conocimiento técnico, sino para ampliar la información que consideraron relevante. Los estudios futuros se orientan al diseño de DD en los que se procura 
que la matemática resulte ser un saber funcional para estudiar situaciones reales, y poder ser gestionados en las condiciones actuales de la escuela secundaria.

\section{Referências}

Barquero, B. Ecología de la modelización matemática en la enseñanza universitaria de las matemáticas. Tesis de doctorado. Universitat Autónoma de Barcelona. 2009.

Chevallard, Y. Vers une didactique de la codisciplinarité. Notes sur une nouvelle épistémologie scolaire. 2004. Disponible en : http://yves.chevallard.free.fr/spip/spip/article.php3?id article $=45$ Acceso: 03 de May. 2018.

Chevallard, Y. Steps towards a new epistemology in mathematics education. In M. Bosch (Ed.), Proceedings of the 4th Conference of the European Society for Research in Mathematics Education. Barcelona: FUNDEMI-IQS, 2006, 21-30.

Chevallard, Y. Passé et présent de la théorie anthropologique du didactique. En L. Ruiz-Higueras, A. Estepa, F. Javier García (Éd.), Sociedad, Escuela y Matemáticas. Aportaciones de la Teoría Antropológica de la Didáctico. Jaén: Universidad de Jaén, 2007, 705- 746.

Chevallard, Y. Enseñar Matemáticas en la Sociedad de Mañana: Alegato a favor de un contraparadigma emergente. REDIMAT, v.2, n.2, 161-182. 2013a.

Chevallard, Y. Journal du Seminaire TAD/IDD. Théorie Anthropologique du Didactique \& Ingénierie Didactique du Développement. 2013b. Disponible en:

http://yves.chevallard.free.fr/spip/spip/IMG/pdf/journal-tad-idd-2009-2010-8.pdf Acceso: 03 de May. 2018.

Chevallard, Y. Éléments de didactique du développement durable. Leçon 3. 2013c. Disponible en: http://yves.chevallard.free.fr/spip/spip/IMG/pdf/Didactique_du_DD_3.pdf

Cid, E., Muñoz, J., Ruíz, N. La introducción de los REI en la formación de profesores: un ejemplo de REI-FP. VI congrès international de la TAD. Autrans, 22 - 26 Enero, 2018. Acceso: 03 de May. 2018.

Corica, A. Enseñanza por Investigación en el marco de la Teoría Antropológica de lo Didáctico: Una propuesta para estudiar las funciones a trozos. Editorial UNCPBA. Tandil. 2016.

Corica, A., Otero, M. Estudio de dispositivos didácticos propuestos por futuros profesores de Matemática: un análisis desde la TAD. Perspectiva Educacional, v.55, n.2, 21-37. 2016a.

Corica, A., Otero, M. (). Diseño e implementación de un curso para la formación de profesores en matemática: una Propuesta desde la TAD. BOLEMA, v.30, n.55, 763-785. 2016 b.

Corica, A., Otero, M. Análisis de un dispositivo didáctico propuesto por futuros profesores de matemática formados en la TAD. AIEM, n.12, 79-95. 2017.

Costa, V. Recorrido de Estudio e Investigación Codisciplinar en la Universidad para la Enseñanza del Cálculo Vectorial en carreras de Ingeniería. Tesis de doctorado. Universidad Nacional del Centro de la Provincia de Buenos Aires. 2013.

Donvito, A., Otero, M. R. y Sureda, P. Actitudes de la pedagogía de la investigación en el marco de la TAD: un análisis en tres escuelas secundarias. Ikastorratza, e-Revista de Didáctica, v.12, 1-27. 2014. 
Fonseca, C. Discontinuidades matemáticas y didácticas entre la Secundaria y la Universidad. Tesis de doctorado. Universidad de Vigo. 2004.

Fonseca, C. Una posible "razón de ser" de la diagonalización de matrices en ciencias económicas y empresariales. En A. Bronner, M. Larguier, M. Artaud, M. Bosch, Y. Chevallard, G. Cirade y C. Ladage (eds.) Diffuser les mathématiques (et les autres savoirs) comme outils de connaissance et d'action. Montpellier: IUFM de Montpellier, 2010, 595-614.

Fonseca, C. Una herramienta para el estudio funcional de las matemáticas: los Recorridos de Estudio e Investigación. Educación Matemática, 23(1), 97-121. 2011a.

Fonseca, C. Los Recorridos de Estudio e Investigación en las escuelas de ingeniería. Educação Matemática Pesquisa São Paulo, v.13, n.3, 547-580. 2011b.

Fonseca, C. Recorridos de Estudio e Investigación: una propuesta dentro de la teoría antropológica de lo didáctico para la creación de secuencias de enseñanza y aprendizaje. Paradigma, v.32, n.1, 55-70. 2011c.

Fonseca, C., Pereira, A. y Casas, J. Los REI en la creación de secuencias de enseñanza y aprendizaje. En M. Bosch, J. Gascón, A. Ruiz Olarría, M. Artaud, A. Bronner, Y. Chevallard, G. Cirade, C. Ladage y M. Larguier (eds.), Un panorama de la TAD. Bellaterra: CRM Centre de Recerca Matemàtica. 2010, 671-684.

Gascón, J. Efectos del autismo temático sobre el estudio de la Geometría en Secundaria II: La clasificación de los cuadriláteros convexos. Suma, v.45, 41-52. 2004.

Hernández, R., Fernández, C.; Baptista, P. Metodología de la investigación. Editorial Mc Graw Hill. México. 2014.

Llanos, V. Enseñanza de la Matemática mediante Recorridos de Estudio e Investigación (REI) en la escuela secundaria: diseño, puesta en aula y análisis de seis implementaciones. Tesis de doctorado. Universidad Nacional del Centro de la Provincia de Buenos Aires. 2012.

Mejía, J. Problemas centrales del análisis de datos cualitativos. Revista Latinoamericana de Metodología de la Investigación Social, v.1, 47-60. 2011.

Oliveira, C. Una posible «razón de ser» del cálculo diferencial elemental en el ámbito de la modelización funcional. Tesis de doctorado. Universidad de Vigo. 2015.

Otero, M. R., Gazzola, M. P., Llanos, V. C. y Arlego, M. Co-disciplinary Physics and Mathematics Research and Study Course (RSC) within Three Study Groups: Teachers-in-training, Secondary School Students and Researchers. Review of Science, Mathematics and ICT Education, v.10, n.2, 55-78. 2016.

Parra, V. Diseño, implementación y evaluación de un REI para el último año del nivel secundario: funcionamiento de las dialécticas. Tesis de doctorado. Universidad Nacional del Centro de la Provincia de Buenos Aires. 2013.

Rodriguez, E. Metacognición, resolución de problemas y enseñanza de las matemáticas. Una propuesta integradora desde el enfoque antropológico. Tesis de doctorado. Universidad Complutense de Madrid. 2005.

Ruiz-Munzón, N. La introducción del álgebra elemental y su desarrollo hacia la modelización funcional. Tesis de doctorado. Universidad Autónoma de Barcelona. 2010.

Ruiz, N., Bosch, M. y Gascón, J. Modelización funcional con parámetros en un taller de matemáticas 
con Wiris. En L. Ruiz Higueras, A. Estepa y F. J. García (eds.), Sociedad, escuela y matemáticas. Aportaciones de la teoría antropológica de lo didáctico. Jaén: Publicaciones de la Universidad de Jaén. 2007, $677-704$.

Ruiz-Higueras, L., García García, F. Análisis de praxeologías didácticas en la gestión de procesos de modelización matemática en la escuela infantil. Relime, v.14, n.1, 41-70. 2011.

Salgado, D., Otero, M., Parra, V. Gestos didácticos en el desarrollo de un recorrido de estudio e investigación en el nivel universitario relativo al cálculo: el funcionamiento de las dialécticas.

Perspectiva Educacional, v.56, n.1, 84-108. 2017.

Serrano, L. La modelización matemática en los estudios universitarios de economía y empresa: análisis ecológico y propuesta didáctica. Tesis de doctorado. Universitat Ramon Llull. 2013.

Serrano, L., Bosch, M., Gascón, J. Cómo hacer una previsión de ventas: propuesta de recorrido de estudio e investigación en un primer curso universitario de administración y dirección de empresas. En A. Bronner, M. Larguier, M. Artaud, M., Bosch, Y. Chevallard, G. Cirade y C. Ladage (eds.), Diffuser les mathématiques (et les autres savoirs) comme outils de connaissance et d'action. Montpellier: IUFM de l'Académie de Montpellier, 2010, 835-857. 